\title{
MS38-P02 | Pushing data quality FOR LABORATORY PAiR Distribution Function
}

\section{EXPERIMENTS}

Zobel, Mirijam (Universität Bayreuth, Bayreuth, GER); Thomä, Sabrina (University Bayreuth, Bayreuth, GER); Prinz, Nils (University Bayreuth, Bayreuth, GER)

Although the very first laboratory X-ray pair distribution function (PDF) measurement was carried out in the 1930s [1], laboratory PDF studies are rare. Only few studies emerged during the last decade [2], but limited $Q_{\max }$ or insufficient instrumental resolution impeded a routine use for structural refinements. Based on a STOE STADI $P$ powder diffractometer in transmission geometry, we designed a novel PDF diffractometer with monochromatic Ag $K \alpha_{1}$ radiation covering a Q-range of $0.3-20.5 \AA^{-1}\left(144^{\circ} 2 \theta\right)$. Four MYTHEN2 silicon strip detectors are arranged as a MYTHEN2 4K module on one detector arm, providing high instrumental resolution and low background. PDF data is collected in a moving mode within 6 hours for powders. For benchmarking, we measured and refined LaB $_{6}$ standards with goodness-of-fits $R_{\mathrm{w}}=0.14$ over $80 \AA$, and ca. $7 \mathrm{~nm} \mathrm{TiO}$, nanoparticles over $30 \AA$ with $R_{\mathrm{w}}=0.18$. While all previous lab PDF studies did not show or refine any data for distances $>30 \AA$ - due to a PDF peak overlap beyond ca. $25 \AA$ due to a lack of monochromatization - we can readily refine our $\mathrm{TiO}_{2}$ PDF data with an $R_{\mathrm{w}}$ as low as 0.22 over $70 \AA$ A. [3] Further data on ionic liquids and metal organic frameworks will be presented.

[1] B.E. Warren, et a., Physical Review 46 (1934) 368

[2] J.T. Nijenhuis, et al., Z. Kristallogr. 2009 (2009) 163

[3] S.L.J. Thomä, et al., Rev. Sci. Instr. (2019), accepted 